\title{
A Concise Route to Benzofused Macrolactones via Ynolides: Cycloproparadicicol
}

\author{
Zhi-Qiang Yang ${ }^{\dagger}$ and Samuel J. Danishefsky ${ }^{*,+, \ddagger}$ \\ ${ }^{\dagger}$ Laboratory for Bioorganic Chemistry, Sloan Kettering Institute for Cancer Research, 1275 York \\ Avenue, New York, New York 10021, and Department of Chemistry, Columbia University, Havemayer \\ Hall, 3000 Broadway, New York, New York 10027
}

Supporting Information Available: Experimental procedure and physical data for all new compounds (PDF). This material is available free of charge via the internet at http://pubs.acs.org. 


\section{Supplementary Material}

General Methods: Reagents obtained from commercial suppliers were used without further purification unless otherwise noted. THF, toluene, and methylene chloride was obtained from a dry solvent system (passed through a prepacked column of alumina) and used without further drying. All air and water

sensitive reactions were performed in oven or flame-dried glassware. NMR $\left({ }^{1} \mathrm{H}\right.$ and $\left.{ }^{13} \mathrm{C}\right)$ spectra were recorded on Bruker AMX-400 MHz or Bruker Advance DRX-500 MHz as noted individually, referenced to $\mathrm{CDCl}_{3}\left(7.27 \mathrm{ppm}\right.$ for ${ }^{1} \mathrm{H}$ and $77.23 \mathrm{ppm}$ for $\left.{ }^{13} \mathrm{C}\right)$. Optical rotations were obtained on a JASCO model DIP-370 digital polarimeter. Low resolution mass spectra (ESI) were determined with a PESciex AP 130 spectrometer. High resolution mass spectra (FAB) were determined at Chemistry Department of Columbia University. Flash chromatography was performed with silica gel (230-400 mesh) from EM Science as the stationary phase. Analytical thin-layer chromatography was performed on E. Merck silica gel 60 F254 plates. Compounds which were not UV active were visualized by dipping the plates in phosphomolybdic acid solution and heating. Preparative thin layer chromatography was performed using the indicated solvent on Whatman ${ }^{\circledR}($ LK6F Silica gel $60 \AA 250 \mu \mathrm{M}$ or Pk6F Silica Gel $60 \AA 1000 \mu \mathrm{M})$ TLC plate.

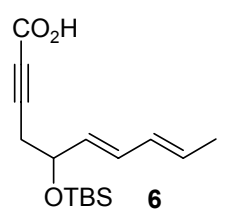

Acid 6. To a suspension of activated zinc $(15 \mathrm{~g}, 230 \mathrm{mmol})$ in dry THF $(50 \mathrm{~mL})$ at $0{ }^{\circ} \mathrm{C}$ was added propargyl bromide $4(19.2 \mathrm{~mL} 80 \mathrm{wt} \%$ in toluene, $172 \mathrm{mmol})$. The resulting mixture was stirred at $0{ }^{\circ} \mathrm{C}$ for $1 \mathrm{hr}$, and sorbaldehyde $5(12.7 \mathrm{~mL}, 115 \mathrm{mmol})$ was added. After $1 \mathrm{hr}$ at $0{ }^{\circ} \mathrm{C}$, additional zinc $(4.5 \mathrm{~g}$, $69 \mathrm{mmol}$ ) was added, and stirring was continued for $2.5 \mathrm{hrs}$ at room temperature (the reaction was exothermic and ice bath was needed occasionally to keep the temperature down). The reaction was 
quenched by slow addition of sat. aqueous $\mathrm{NH}_{4} \mathrm{Cl}(500 \mathrm{~mL})$, followed by diluting with $\mathrm{Et}_{2} \mathrm{O}(1 \mathrm{~L})$. The layers were separated, and the organic layer was washed with $\mathrm{H}_{2} \mathrm{O}(300 \mathrm{~mL})$, brine $(300 \mathrm{~mL})$, dried $\left(\mathrm{Na}_{2} \mathrm{SO}_{4}\right)$, filtered and concentrated in vacuum. The residue was dissolved in $\mathrm{CH}_{2} \mathrm{Cl}_{2}(750 \mathrm{~mL})$ with imidazole (9.8 g, $144 \mathrm{mmol}), t$-butyldimethylsilyl chloride (19 g, $126 \mathrm{mmol})$ and 4-(dimethylamino) pyridine $(1.4 \mathrm{~g}, 11.5 \mathrm{mmol})$, and stirred at room temperature for $3 \mathrm{hrs}$. Additional imidazole (4.9 g, 72 mmol) and $t$-butyldimethylsilyl chloride $(9.5 \mathrm{~g}, 63 \mathrm{mmol})$ were added, and stirring was continued for 9 hrs. The reaction was quenched by addition of sat. aqueous $\mathrm{NH}_{4} \mathrm{Cl}(200 \mathrm{~mL})$. The layers were separated, and the organic layer was washed with $\mathrm{H}_{2} \mathrm{O}(200 \mathrm{~mL})$, brine $(200 \mathrm{~mL})$, dried $\left(\mathrm{Na}_{2} \mathrm{SO}_{4}\right)$, filtered and concentrated in vacuum. The residue was purified by flash chromatography (silica, 0 to $10 \% \mathrm{Et}_{2} \mathrm{O}$ in hexane) to give the terminal alkyne precursor of $6(15 \mathrm{~g}, 52 \%) .{ }^{1} \mathrm{H} \mathrm{NMR}\left(\mathrm{CDCl}_{3}, 400 \mathrm{MHz}\right) \delta 6.18(\mathrm{dd}, J$ $=15.1,10.5 \mathrm{~Hz}, 1 \mathrm{H}), 6.03(\mathrm{ddd}, J=15.0,10.6,1.3 \mathrm{~Hz}, 1 \mathrm{H}), 5.72(\mathrm{dd}, J=14.9,6.9 \mathrm{~Hz}, 1 \mathrm{H}), 5.61(\mathrm{dd}, J$ $=15.1,6.4 \mathrm{~Hz}, 1 \mathrm{H}), 4.30(\mathrm{q}, J=6.3 \mathrm{~Hz}, 1 \mathrm{H}), 2.43(\mathrm{ddd}, \mathrm{J}=16.5,6.2,2.7 \mathrm{~Hz}, 1 \mathrm{H}), 2.34(\mathrm{ddd}, \mathrm{J}=16.5$, 6.8, 1.7 Hz, 1H), $2.60(\mathrm{t}, J=2.6 \mathrm{~Hz}, 1 \mathrm{H}), 1.77(\mathrm{~d}, J=6.8 \mathrm{~Hz}, 3 \mathrm{H}), 0.91(\mathrm{~s}, 9 \mathrm{H}), 0.09,0.06(2 \mathrm{~s}, 6 \mathrm{H}) ;{ }^{13} \mathrm{C}$ $\operatorname{NMR}\left(\mathrm{CDCl}_{3}, 100 \mathrm{~Hz}\right) \delta 132.5,131.1,130.7,130.0,81.7,72.1,70.1,28.9,26.1,18.4,-2.7$; IR (film) $v_{\max } 3313,2956,2930,2856,2121,1255,1115,1079,987,837 ;$ ESIMS $m / z 273\left(\left[\mathrm{M}+\mathrm{Na}^{+}\right]\right.$ $\mathrm{C}_{15} \mathrm{H}_{26} \mathrm{NaOSi}$ requires 273).

To a solution of the terminal alkyne precursor of $6(15.0 \mathrm{~g}, 59.9 \mathrm{mmol})$ in $\mathrm{Et}_{2} \mathrm{O}(270 \mathrm{~mL})$ at $-78{ }^{\circ} \mathrm{C}$, was added a solution of BuLi (1.6 M in hexane, $41.5 \mathrm{~mL}, 66.4 \mathrm{mmol})$. After $45 \mathrm{~min}$, excess crushed dry ice was added and the reaction was allowed to warm to room temperature. The solution was acidified by addition of $0.5 \mathrm{M}$ aqueous citric acid $(300 \mathrm{~mL})$. The layers were separated, and the aqueous layer was extracted with additional $\mathrm{Et}_{2} \mathrm{O}\left(300 \mathrm{~mL}\right.$ x 2). The combined organic layers were dried $\left(\mathrm{MgSO}_{4}\right)$, filtered and concentrated in vacuum. The residue was purified by flash chromatography (silica, $50 \%$ to $100 \%$ EtOAc in hexane) to give the product as a light yellow solid (17.5 g, 99\%). ${ }^{1} \mathrm{H} \mathrm{NMR}\left(\mathrm{CDCl}_{3}, 400 \mathrm{MHz}\right)$ $\delta 6.19(\mathrm{dd}, J=15.1,10.4 \mathrm{~Hz}, 1 \mathrm{H}), 6.03(\mathrm{ddd}, J=14.9,10.6,1.3 \mathrm{~Hz}, 1 \mathrm{H}), 5.75(\mathrm{dd}, J=15.0,6.8 \mathrm{~Hz}$, 1H), $5.55(\mathrm{dd}, J=15.1,6.4 \mathrm{~Hz}, 1 \mathrm{H}), 4.36(\mathrm{q}, J=6.3 \mathrm{~Hz}, 1 \mathrm{H}), 2.61-2.48(\mathrm{~m}, 2 \mathrm{H}), 1.77(\mathrm{~d}, J=6.8 \mathrm{~Hz}$, 
3H), $0.91(\mathrm{~s}, 9 \mathrm{H}), 0.10,0.06(2 \mathrm{~s}, 6 \mathrm{H}) ;{ }^{13} \mathrm{C} \mathrm{NMR}\left(\mathrm{CDCl}_{3}, 100 \mathrm{~Hz}\right) \delta 157.6,131.6,131.3,130.7,89.7$, 74.0, 71.4, 29.3, 26.0, 18.4, 18.3, -4.3, -4.7; IR (film) $v_{\max } 2956,2930,2857,2242,1689,1281,1257$, 1080; ESIMS $m / z 317\left(\left[\mathrm{M}+\mathrm{Na}^{+}\right], \mathrm{C}_{16} \mathrm{H}_{26} \mathrm{NaO}_{3}\right.$ Si requires 317).

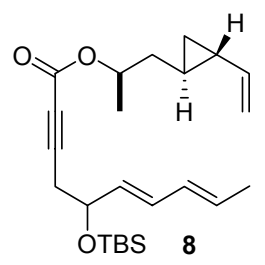

Alkynoic ester 8. To a solution of DIAD $(14.7 \mathrm{~mL}, 72.9 \mathrm{mmol})$ in dry THF $(350 \mathrm{~mL})$ was added $\mathrm{Ph}_{3} \mathrm{P}$ (15.8 g, $60.2 \mathrm{mmol})$, and the mixture was stirred at room temperature for one hour. At $-20^{\circ} \mathrm{C}$, a solution of acid 6 (13.1 g, $44.4 \mathrm{mmol})$ in $100 \mathrm{~mL}$ THF was added. After $15 \mathrm{~min}$, a solution of alcohol 7 (4.0 g, $31.7 \mathrm{mmol}$ ) in $150 \mathrm{~mL}$ THF was added, and stirring was continued for 2 hours at $-20{ }^{\circ} \mathrm{C}$. The reaction was quenched by addition of $250 \mathrm{~mL}$ of $\mathrm{pH} 7.2$ phosphate buffer, followed by warming to room temperature and diluting with EtOAc $(1.5 \mathrm{~L})$. The layers were separated, and the aqueous layer was extracted with EtOAc $(2 \times 250 \mathrm{~mL})$. The combined organic layers were washed with brine $(250 \mathrm{~mL})$, dried $\left(\mathrm{Na}_{2} \mathrm{SO}_{4}\right)$, filtered and concentrated in vacuum. The residue was purified by flash chromatography (silica, 50:1 $\rightarrow 20: 1$ hexanes/EtOAc) to give ester $\mathbf{8}$ as a mixture of two inseparable diastereoisomers (5.9 g, 47\%). ${ }^{1} \mathrm{H} \mathrm{NMR}\left(\mathrm{CDCl}_{3}, 400 \mathrm{MHz}\right) \delta 6.17(\mathrm{dd}, J=15.1,10.5 \mathrm{~Hz}, 1 \mathrm{H}), 6.03(\mathrm{ddd}, J=12.3,10.6,1.4$ $\mathrm{Hz}, 1 \mathrm{H}), 5.70(\mathrm{dd}, J=14.8,6.8 \mathrm{~Hz}, 1 \mathrm{H}), 5.55(\mathrm{dd}, J=15.2,6.4 \mathrm{~Hz}, 1 \mathrm{H}), 5.37$ (ddd, $J=17.1,10.2,8.7$ $\mathrm{Hz}, 1 \mathrm{H}), 5.06(\mathrm{q}, J=6.4 \mathrm{~Hz}, 1 \mathrm{H}), 5.03(\mathrm{dd}, J=17.0,1.5 \mathrm{~Hz}, 1 \mathrm{H}), 4.84(\mathrm{dd}, J=10.2,1.6 \mathrm{~Hz}, 1 \mathrm{H}), 4.34$ (q, $J=6.4 \mathrm{~Hz}, 1 \mathrm{H}), 2.56-2.42(\mathrm{~m}, 2 \mathrm{H}), 1.76(\mathrm{~d}, J=6.8 \mathrm{~Hz}, 3 \mathrm{H}), 1.57-1.53(\mathrm{~m}, 2 \mathrm{H}), 1.29(\mathrm{~d}, J=6.4 \mathrm{~Hz}$, $3 \mathrm{H}), 1.20-1.10(\mathrm{~m}, 1 \mathrm{H}), 0.90(\mathrm{~s}, 9 \mathrm{H}), 0.79-0.68(\mathrm{~m}, 1 \mathrm{H}), 0.65-0.57(\mathrm{~m}, 2 \mathrm{H}), 0.10,0.05(2 \mathrm{~s}, 6 \mathrm{H}) ;{ }^{13} \mathrm{C}$ $\operatorname{NMR}\left(\mathrm{CDCl}_{3}, 100 \mathrm{~Hz}\right) \delta 154.5,141.4,131.8,131.1,130.8,130.4,112.1,86.2,74.9,73.0,71.5,39.7$, 29.2, 26.0, 22.4, 19.8, 18.4, 18.3, 17.2, 13.7, -4.3, -4.7; IR (film) $v_{\max } 2955,2930,2856,2238,1710$, 
1253, 1068; ESIMS m/z $437\left(\left[\mathrm{M}+\mathrm{Cl}^{-}\right], \mathrm{C}_{24} \mathrm{H}_{38} \mathrm{ClO}_{3}\right.$ Si requires 437); HRMS $\left(\mathrm{FAB}^{+}\right) \mathrm{m} / z 403.2687$ ([M $+\mathrm{H}]^{+}, \mathrm{C}_{24} \mathrm{H}_{39} \mathrm{O}_{3} \mathrm{Si}$ requires 403.2668).

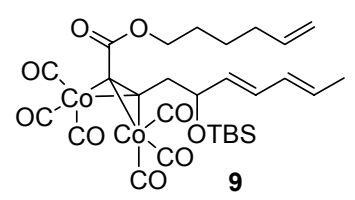

Cobalt complex 9. To a solution of acid 6 (192 mg, $0.653 \mathrm{mmol})$ and 5-hexen-1-ol (0.118 mL, 0.979 mmol) in dry $\mathrm{CH}_{2} \mathrm{Cl}_{2}(3 \mathrm{~mL})$ was added EDCI (150 mg, $\left.0.784 \mathrm{mmol}\right)$ and 4-(dimethylamino)pyridine (8.0 mg, $0.065 \mathrm{mmol})$. After $3 \mathrm{hrs}$ at room temperature, the reaction mixture was loaded on PTLC plates and purified (12:1 hexane/EtOAc) to give the model ester (146 mg, 59\%). ${ }^{1} \mathrm{H} \mathrm{NMR}\left(\mathrm{CDCl}_{3}, 400 \mathrm{MHz}\right)$ $\delta 6.18(\mathrm{dd}, J=15.1,10.5 \mathrm{~Hz}, 1 \mathrm{H}), 6.04(\mathrm{ddd}, J=15.0,10.5,1.5 \mathrm{~Hz}, 1 \mathrm{H}), 5.79$ (ddt, $J=17.1,10.3,7.2$ $\mathrm{Hz}, 1 \mathrm{H}), 5.71(\mathrm{dq}, J=15.0,6.8 \mathrm{~Hz}, 1 \mathrm{H}), 5.56(\mathrm{dd}, J=15.1,6.4 \mathrm{~Hz}, 1 \mathrm{H}), 5.03(\mathrm{dq}, J=17.1,1.6 \mathrm{~Hz}, 1 \mathrm{H})$, $4.97(\mathrm{dd}, J=10.2,1.6 \mathrm{~Hz}, 1 \mathrm{H}), 4.35(\mathrm{q}, J=6.3 \mathrm{~Hz}, 1 \mathrm{H}), 4.16(\mathrm{t}, J=6.6 \mathrm{~Hz}, 2 \mathrm{H}), 2.57-2.44(\mathrm{~m}, 2 \mathrm{H})$, $2.09(\mathrm{q}, J=7.2 \mathrm{~Hz}, 1 \mathrm{H}), 1.77(\mathrm{~d}, J=6.9 \mathrm{~Hz}, 3 \mathrm{H}), 1.74-1.63(\mathrm{~m}, 2 \mathrm{H}), 1.52-1.44(\mathrm{~m}, 2 \mathrm{H}), 0.90(\mathrm{~s}, 9 \mathrm{H})$, $0.10,0.06(2 \mathrm{~s}, 6 \mathrm{H}) ;{ }^{13} \mathrm{C} \mathrm{NMR}\left(\mathrm{CDCl}_{3}, 100 \mathrm{~Hz}\right) \delta 154.0,138.4,131.8,131.1,130.8,130.5,115.1,86.6$ $74.6,71.5,65.9,33.4,29.3,28.1,26.0,25.3,18.4,18.3,-4.3,-4.7$; IR (film) $v_{\max } 2955,2930,2856$, 2238, 1713, 1249, 1072; ESIMS $m / z 399\left(\left[\mathrm{M}+\mathrm{Na}^{+}\right], \mathrm{C}_{22} \mathrm{H}_{36} \mathrm{NaO}_{3}\right.$ Si requires 399). HRMS (FAB $\left.{ }^{+}\right) m / z$ $375.2363\left([\mathrm{M}-\mathrm{H}]^{+}, \mathrm{C}_{22} \mathrm{H}_{35} \mathrm{O}_{3} \mathrm{Si}\right.$ requires 375.2355).

To a solution of the above alkynoic ester $(77.8 \mathrm{mg}, 0.207 \mathrm{mmol})$ in toluene $(9 \mathrm{~mL})$ was added $\mathrm{Co}_{2}(\mathrm{CO})_{8}$ (99.0 mg, $0.289 \mathrm{mmol}$ ). The mixture was stirred at room temperature for $45 \mathrm{~min}$, and then concentrated in vacuum. The dark residue was purified by PTLC (15:1 hexane/EtOAc) to give cobalt complex 9 (117.5 mg, 86\%) as a red oil. ${ }^{1} \mathrm{H} \mathrm{NMR}\left(\mathrm{CDCl}_{3}, 400 \mathrm{MHz}\right) \delta 6.17(\mathrm{dd}, J=15.3,10.6 \mathrm{~Hz}, 1 \mathrm{H}), 6.03(\mathrm{ddd}$, $J=15.3,11.3 \mathrm{~Hz}, 1 \mathrm{H}), 5.68(\mathrm{dd}, J=14.9,6.9 \mathrm{~Hz}, 1 \mathrm{H}), 5.61(\mathrm{dd}, J=15.2,6.8 \mathrm{~Hz}, 1 \mathrm{H}), 5.38(\mathrm{ddd}, J=$ $17.1,10.1,8.7 \mathrm{~Hz}, 1 \mathrm{H}), 5.10(\mathrm{q}, J=6.4 \mathrm{~Hz}, 1 \mathrm{H}), 5.04(\mathrm{dd}, J=17.1,1.3 \mathrm{~Hz}, 1 \mathrm{H}), 4.85(\mathrm{dd}, J=10.3,1.4$ $\mathrm{Hz}, 1 \mathrm{H}), 4.41,(\mathrm{~m}, 1 \mathrm{H}), 3.20-3.15(\mathrm{~m}, 2 \mathrm{H}), 1.76(\mathrm{~d}, J=6.7 \mathrm{~Hz}, 3 \mathrm{H}), 1.59(\mathrm{t}, J=6.6 \mathrm{~Hz}, 2 \mathrm{H}), 1.32(\mathrm{~d}, J=$ 
$6.2 \mathrm{~Hz}, 3 \mathrm{H}), 1.22-1.17(\mathrm{~m}, 1 \mathrm{H}), 0.90(\mathrm{~s}, 9 \mathrm{H}), 0.82-0.72(\mathrm{~m}, 1 \mathrm{H}), 0.66-0.59(\mathrm{~m}, 2 \mathrm{H}), 0.09,0.08(2 \mathrm{~s}, 6 \mathrm{H})$;

${ }^{13} \mathrm{C} \mathrm{NMR}\left(\mathrm{CDCl}_{3}, 100 \mathrm{~Hz}\right) \delta 198.7,169.2,141.4,134.3,132.5,132.4,132.2,131.0,130.2,128.8,127.2$ $127.1,112.0,93.0,81.0,73.7,73.6,73.1,40.0,26.1,22.4,19.9,18.6,18.4,17.4,13.9,13.5,-4.2,-4.3,-$ 4.6; IR (film) $v_{\max } 2956,2930,2858,2097,2058,2029,1703,1221,1065 ;$ ESIMS $m / z 685\left(\left[\mathrm{M}+\mathrm{Na}^{+}\right]\right.$, $\mathrm{C}_{28} \mathrm{H}_{36} \mathrm{Co}_{2} \mathrm{NaO}_{9} \mathrm{Si}$ requires 685).

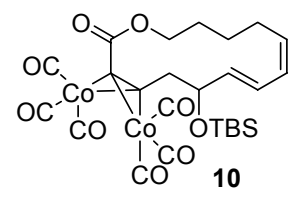

RCM product 10. To a solution of cobalt complex 9 (16 mg, $0.024 \mathrm{mmol})$ in dry $\mathrm{CH}_{2} \mathrm{Cl}_{2}(120 \mathrm{~mL})$ was added tricyclohexyl phosphine[1,3-bis(2,4,6-trimethylphenyl)-4,5-dihydroimidazol-2-ylidene]-[bezylidene] ruthenium(IV) dichloride (second generation Grubbs catalyst) (6.1 mg, $0.0072 \mathrm{mmol})$. The resulting solution was heated to $45^{\circ} \mathrm{C}$ for $1 \mathrm{hr}$ and $10 \mathrm{~min}$, then cooled to room temperature and filtered through a plug of silica gel. The solvent was removed under reduced pressure. The residue was purified by PTLC (15:1 hexane/EtOAc) to give cyclic product $10(10.5 \mathrm{mg}, 71 \%) .{ }^{1} \mathrm{H} \mathrm{NMR}\left(\mathrm{CDCl}_{3}, 400 \mathrm{MHz}\right) \delta$ $6.44(\mathrm{dd}, J=15.3,10.8 \mathrm{~Hz}, 1 \mathrm{H}), 5.97(\mathrm{t}, J=10.8 \mathrm{~Hz}, 1 \mathrm{H}), 5.58(\mathrm{dd}, J=15.4,7.5 \mathrm{~Hz}, 1 \mathrm{H}), 5.54(\mathrm{dt}, J=$ $10.1,4.5 \mathrm{~Hz}, 1 \mathrm{H}), 4.57-4.47(\mathrm{~m}, 2 \mathrm{H}), 4.25-4.20(\mathrm{~m}, 1 \mathrm{H}), 3.45-3.36(\mathrm{~m}, 2 \mathrm{H}), 2.45-2.37(\mathrm{~m}, 1 \mathrm{H}), 2.13-$ $1.05(\mathrm{~m}, 1 \mathrm{H}), 1.99-1.79(\mathrm{~m}, 1 \mathrm{H}), 1.80-1.62(\mathrm{~m}, 2 \mathrm{H}), 1.51-1.41(\mathrm{~m}, 1 \mathrm{H}), 0.92(\mathrm{~s}, 9 \mathrm{H}), 0.11,0.08(2 \mathrm{~s}, 6 \mathrm{H})$ ${ }^{13} \mathrm{C} \mathrm{NMR}\left(\mathrm{CDCl}_{3}, 100 \mathrm{~Hz}\right) \delta 199.9,170.4,135.2,132.7,129.0,127.1,92.8,77.4,73.1,65.0,45.2,26.4$, 26.1, 25.4, 18.5, 1.2, -4.2, -4.6; IR (film) $v_{\max } 2955,2930,2857,2098,2059,2027,1702,1213,1057$; ESIMS $m / z 643\left(\left[\mathrm{M}+\mathrm{Na}^{+}\right], \mathrm{C}_{25} \mathrm{H}_{30} \mathrm{Co}_{2} \mathrm{NaO}_{9}\right.$ Si requires 643).

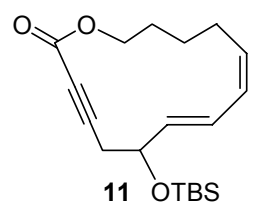


Model cyclic alkyne 11. To a solution of compound $\mathbf{1 0}(35.6 \mathrm{mg}, 0.0574 \mathrm{mmol})$ in acetone at $-10{ }^{\circ} \mathrm{C}$ was added ammonium cerium (IV) nitrate (189 $\mathrm{mg}, 0.344 \mathrm{mmol})$ portionwise. After $10 \mathrm{~min}$ at $-10{ }^{\circ} \mathrm{C}$, the reaction was quenched by addition of diisopropylethylamine $(0.18 \mathrm{~mL}, 1.03 \mathrm{mmol})$. The resulting mixture was filtered through a plug of neutral alumina, and the solvent was removed under reduced pressure. Purification by PTLC (15:1 hexane/EtOAc) afforded cyclic alkyne 11 (17.6 mg, 92\%). ${ }^{1} \mathrm{H}$ $\operatorname{NMR}\left(\mathrm{CDCl}_{3}, 400 \mathrm{MHz}\right) \delta 6.64(\mathrm{dd}, J=15.5,11.1 \mathrm{~Hz}, 1 \mathrm{H}), 6.07(\mathrm{t}, J=11.0 \mathrm{~Hz}, 1 \mathrm{H}), 5.53(\mathrm{dd}, J=$ 15.5, 7.1 Hz, 1H), 5.40 (dt, $J=10.4,4.8 \mathrm{~Hz}, 1 \mathrm{H})$, ), 4.41-4.25 (m, 2H), 4.06-4.01 (m, 1H), 2.69-2.62 (m, 1H), $2.56(\mathrm{dd}, J=17.1,4.4 \mathrm{~Hz}, 1 \mathrm{H}), 2.46(\mathrm{dd}, J=17.1,9.5 \mathrm{~Hz}, 1 \mathrm{H}), 2.26-2.21(\mathrm{~m}, 1 \mathrm{H}), 1.75-1.61$ (m, 4H), $0.89(\mathrm{~s}, 9 \mathrm{H}), 0.08,0.07(2 \mathrm{~s}, 6 \mathrm{H}) ;{ }^{13} \mathrm{C} \mathrm{NMR}\left(\mathrm{CDCl}_{3}, 100 \mathrm{~Hz}\right) \delta 153.9,133.2,132.9,128.5,128.2$ $87.7,77.0,72.9,68.0,29.0,28.2,26.7,26.0,25.6,18.3,-4.3,-4.7$; IR (film) $v_{\max } 2954,2929,2857$, 2238, 1716, 1245, 1110, 1075, 837; ESIMS m/z $357\left(\left[\mathrm{M}+\mathrm{Na}^{+}\right], \mathrm{C}_{19} \mathrm{H}_{30} \mathrm{NaO}_{3} \mathrm{Si}\right.$ requires 357). $\operatorname{HRMS}\left(\mathrm{FAB}^{+}\right) \mathrm{m} / z 333.1888\left([\mathrm{M}-\mathrm{H}]^{+}, \mathrm{C}_{19} \mathrm{H}_{29} \mathrm{O}_{3} \mathrm{Si}\right.$ requires 333.1886).

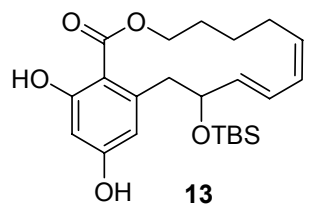

Diels-Alder product 13. Cyclic alkyne $11(27 \mathrm{mg}, 0.081 \mathrm{mmol})$ and excess diene $12(0.30 \mathrm{~mL}, 0.90$ mmol) were mixed and heated in a sealed vial to $140^{\circ} \mathrm{C}$ for 48.5 hours. The mixture was cooled to room temperature, loaded onto a PTLC plate, and purified (4:1 hexane/EtOAc) to afford aromatic product 13 (20 mg, 60\%). ${ }^{1} \mathrm{H} \mathrm{NMR}\left(\mathrm{CDCl}_{3}, 400 \mathrm{MHz}\right) \delta 11.64,(\mathrm{~s}, 1 \mathrm{H}), 6.38(\mathrm{dd}, J=15.4,10.9 \mathrm{~Hz}, 1 \mathrm{H}), 6.35(\mathrm{~d}, J$ $=2.6 \mathrm{~Hz}, 1 \mathrm{H}), 6.30(\mathrm{~d}, J=2.6 \mathrm{~Hz}, 1 \mathrm{H}), 6.23(\mathrm{t}, J=10.6 \mathrm{~Hz}, 1 \mathrm{H}), 5.95(\mathrm{~s}, 1 \mathrm{H}), 5.78(\mathrm{dd}, J=15.3,8.4$ $\mathrm{Hz}, 1 \mathrm{H}), 5.60(\mathrm{q}, J=9.9 \mathrm{~Hz}, 1 \mathrm{H}), 4.69(\mathrm{q}, J=9.1 \mathrm{~Hz}, 1 \mathrm{H}), 4.12(\mathrm{t}, J=8.5 \mathrm{~Hz}, 2 \mathrm{H}), 3.63(\mathrm{~d}, J=13.0 \mathrm{~Hz}$ $1 \mathrm{H}), 2.62(\mathrm{dd}, J=13.1,8.9 \mathrm{~Hz}, 1 \mathrm{H}), 2.50-2.40(\mathrm{~m}, 1 \mathrm{H}), 2.12-2.05(\mathrm{~m}, 1 \mathrm{H}), 1.87-1.76(\mathrm{~m}, 2 \mathrm{H}), 1.56-$ $1.44(\mathrm{~m}, 2 \mathrm{H}), 0.78(\mathrm{~s}, 9 \mathrm{H}),-0.20,-0.25(2 \mathrm{~s}, 6 \mathrm{H}) ;{ }^{13} \mathrm{C} \mathrm{NMR}\left(\mathrm{CDCl}_{3}, 100 \mathrm{~Hz}\right) \delta 172.2,165.7,160.3$ $144.8,135.4,131.0,129.9,126.0,113.0,105.3,102.2,78.7,64.3,46.6,25.9,24.2,23.7,23.0,18.4,-4.7$ 
-5.0; IR (film) $v_{\max } 3380,2954,2929,2856,1648,1620,1254,1169,1106,1061,837$; ESIMS $m / z 441$ $\left(\left[\mathrm{M}+\mathrm{Na}^{+}\right], \mathrm{C}_{23} \mathrm{H}_{34} \mathrm{NaO}_{5} \mathrm{Si}\right.$ requires 441). HRMS $\left(\mathrm{FAB}^{+}\right) \mathrm{m} / z 418.2173\left([\mathrm{M}]^{+}, \mathrm{C}_{23} \mathrm{H}_{34} \mathrm{O}_{5} \mathrm{Si}\right.$ requires 418.2176).

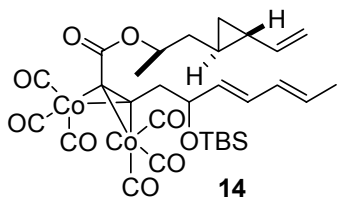

Cobalt complex 14. To a solution of alkyne $8(526 \mathrm{mg}, 1.31 \mathrm{mmol})$ in toluene $(60 \mathrm{~mL})$ was added $\mathrm{Co}_{2}(\mathrm{CO})_{8}(625 \mathrm{mg}, 1.83 \mathrm{mmol})$. The mixture was stirred at room temperature for $30 \mathrm{~min}$, and the solvent was removed under reduced pressure. The dark residue was purified by flash chromatography ( silica, 0 to 5\% EtOAc in hexane) to give cobalt complex 14 (902 $\mathrm{mg}, 100 \%)$ as an inseparable mixture of two diastereomers. ${ }^{1} \mathrm{H} \mathrm{NMR}\left(\mathrm{CDCl}_{3}, 400 \mathrm{MHz}\right) \delta 6.17(\mathrm{dd}, J=15.3,10.6 \mathrm{~Hz}, 1 \mathrm{H}), 6.03(\mathrm{ddd}, J=$ $15.3,11.3 \mathrm{~Hz}, 1 \mathrm{H}), 5.68(\mathrm{dd}, J=14.9,6.9 \mathrm{~Hz}, 1 \mathrm{H}), 5.61(\mathrm{dd}, J=15.2,6.8 \mathrm{~Hz}, 1 \mathrm{H}), 5.38(\mathrm{ddd}, J=17.1$, $10.1,8.7 \mathrm{~Hz}, 1 \mathrm{H}), 5.10(\mathrm{q}, J=6.4 \mathrm{~Hz}, 1 \mathrm{H}), 5.04(\mathrm{dd}, J=17.1,1.3 \mathrm{~Hz}, 1 \mathrm{H}), 4.85(\mathrm{dd}, J=10.3,1.4 \mathrm{~Hz}$, $1 \mathrm{H}), 4.41,(\mathrm{~m}, 1 \mathrm{H}), 3.20-3.15(\mathrm{~m}, 2 \mathrm{H}), 1.76(\mathrm{~d}, J=6.7 \mathrm{~Hz}, 3 \mathrm{H}), 1.59(\mathrm{t}, J=6.6 \mathrm{~Hz}, 2 \mathrm{H}), 1.32(\mathrm{~d}, J=6.2$ $\mathrm{Hz}, 3 \mathrm{H}), 1.22-1.17(\mathrm{~m}, 1 \mathrm{H}), 0.90(\mathrm{~s}, 9 \mathrm{H}), 0.82-0.72(\mathrm{~m}, 1 \mathrm{H}), 0.66-0.59(\mathrm{~m}, 2 \mathrm{H}), 0.09,0.08(2 \mathrm{~s}, 6 \mathrm{H}) ;{ }^{13} \mathrm{C}$ $\mathrm{NMR}\left(\mathrm{CDCl}_{3}, 100 \mathrm{~Hz}\right) \delta 198.7,169.2,141.4,134.3,132.5,132.4,132.2,131.0,130.2,128.8,127.2$ $127.1,112.0,93.0,81.0,73.7,73.6,73.1,40.0,26.1,22.4,19.9,18.6,18.4,17.4,13.9,13.5,-4.2,-4.3,-$ 4.6; IR (film) $v_{\max } 2956,2930,2858,2097,2058,2029,1703,1221,1065$; ESIMS m/z $711\left(\left[\mathrm{M}+\mathrm{Na}^{+}\right]\right.$, $\mathrm{C}_{30} \mathrm{H}_{38} \mathrm{Co}_{2} \mathrm{NaO}_{9} \mathrm{Si}$ requires 711).

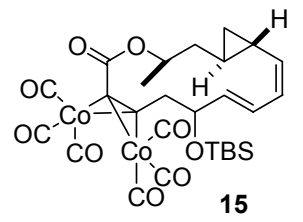

RCM product 15. To a solution of alkyne-cobalt complex $14(67 \mathrm{mg}, 0.097 \mathrm{mmol})$ in $\operatorname{dry} \mathrm{CH}_{2} \mathrm{Cl}_{2}(485$ $\mathrm{mL}$ ) was added tricyclohexyl phosphine[1,3-bis(2,4,6-trimethylphenyl)-4,5-dihydroimidazol-2-ylidene] 
[bezylidene] ruthenim(IV) dichloride (second generation Grubbs catalyst) $(21 \mathrm{mg}, 0.025 \mathrm{mmol})$. The resulting mixture was heated to $45^{\circ} \mathrm{C}$ for 1.5 hours, and filtered through a short column of silica gel. The filtrate was concentrated in vacuum. The residue was purified by PTLC (15:1 hexanes/EtOAc) to give cyclic product 15 as a 2:1 mixture of two separable diastereomers. Major isomer (23.1 $\mathrm{mg}, 37 \%)$ : ${ }^{1} \mathrm{H}$ NMR $\left(\mathrm{CDCl}_{3}, 400 \mathrm{MHz}\right) \delta 6.49(\mathrm{dd}, J=15.4,10.9 \mathrm{~Hz}, 1 \mathrm{H}), 5.84(\mathrm{t}, J=10.6 \mathrm{~Hz}, 1 \mathrm{H}), 5.50(\mathrm{dd}, J=$ 15.4, $8.5 \mathrm{~Hz}, 1 \mathrm{H}), 5.06(\mathrm{dd}, J=10.5,6.9 \mathrm{~Hz}, 1 \mathrm{H}), 5.02-4.94(\mathrm{~m}, 1 \mathrm{H}), 4.81-4.75(\mathrm{~m}, 1 \mathrm{H}) .3 .48-3.36(\mathrm{~m}$, 2H), 2.22-2.25 (m, 1H), 1.58-1.50 (m, 1H), 1.50-1.46 (m, 1H), 1.32 (d, J=6.2 Hz, 3H), $0.91(\mathrm{~s}, 9 \mathrm{H})$, $0.90-0.80(\mathrm{~m}, 1 \mathrm{H}), 0.89$ (s, 9H), 0.67-0.63 (m, 1H), 0.59-0.55 (m, 1H), 0.12, $0.09(2 \mathrm{~s}, 6 \mathrm{H}) ;{ }^{13} \mathrm{C}$ NMR $\left(\mathrm{CDCl}_{3}, 100 \mathrm{~Hz}\right) \delta 199.0,170.4,135.7,134.9,129.2,127.8,92.0,77.5,72.5,72.1,45.5,38.0,26.1,10.3$, 18.4, 16.4, 16.0, -4.2, -4.6; IR (film) $v_{\max } 2955,2928,2854,2097,2060,2029,1692,1232,1056$; ESIMS $m / z 669\left(\left[\mathrm{M}+\mathrm{Na}^{+}\right], \mathrm{C}_{27} \mathrm{H}_{32} \mathrm{Co}_{2} \mathrm{NaO}_{9} \mathrm{Si}\right.$ requires 669); $[\alpha]^{25}-127\left(c 0.11, \mathrm{CHCl}_{3}\right)$. Minor isomer (12.6 mg, 20\%): ${ }^{1} \mathrm{H} \mathrm{NMR}\left(\mathrm{CDCl}_{3}, 500 \mathrm{MHz}\right) \delta 6.60(\mathrm{dd}, J=15.7,9.9 \mathrm{~Hz}, 1 \mathrm{H}), 5.86(\mathrm{t}, J=10.3 \mathrm{~Hz}$, 1H), $5.81(\mathrm{dd}, J=15.7,4.4 \mathrm{~Hz}, 1 \mathrm{H}), 5.12-5.05(\mathrm{~m}, 1 \mathrm{H}), 4.99(\mathrm{t}, J=9.9 \mathrm{~Hz}, 1 \mathrm{H}), 4.46-4.44(\mathrm{~m}, 1 \mathrm{H}), 3.58$ (dd, $J=15.3,9.4 \mathrm{~Hz}, 1 \mathrm{H}), 3.32(\mathrm{dd}, J=15.3,4.2 \mathrm{~Hz}, 1 \mathrm{H}), 2.16(\mathrm{dt}, J=15.3,4.2 \mathrm{~Hz}, 1 \mathrm{H}), 1.65-1.59(\mathrm{~m}$, $2 \mathrm{H}), 1.36(\mathrm{~d}, J=6.5 \mathrm{~Hz}, 3 \mathrm{H}), 0.94(\mathrm{~s}, 9 \mathrm{H}), 0.64-0.59(\mathrm{~m}, 2 \mathrm{H}), 0.14(\mathrm{~s}, 6 \mathrm{H}) ;{ }^{13} \mathrm{C} \mathrm{NMR}\left(\mathrm{CDCl}_{3}, 125 \mathrm{~Hz}\right)$ $\delta 198.6,169.7,135.9,132.6,126.8,126.5,92.0,80.7,73.4,73.0,41.8,37.7,26.1,19.3,18.5,18.4,15.1$, 13.7, -4.5, -4.6; IR (film) $v_{\max } 2929,2856,2097,2059,2028,1702,1220,1059$; ESIMS m/z 669 ([M + $\left.\mathrm{Na}^{+}\right], \mathrm{C}_{27} \mathrm{H}_{32} \mathrm{Co}_{2} \mathrm{NaO}_{9} \mathrm{Si}$ requires 669). $[\alpha]^{25}+48\left(c 0.19, \mathrm{CHCl}_{3}\right)$.

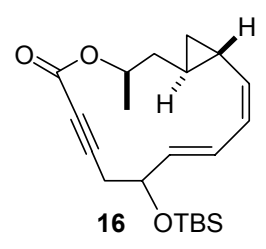

Cyclic alkyne 16. The major isomer of $\mathbf{1 5}(23.1 \mathrm{mg}, 0.0358 \mathrm{mmol})$ was dissolved in dry THF $(1 \mathrm{~mL})$. At $0{ }^{\circ} \mathrm{C}$, a solution of $\mathrm{I}_{2}(135 \mathrm{mg}, 0.536 \mathrm{mmol})$ in THF $(5 \mathrm{~mL})$ was added. After 35 minutes at $0{ }^{\circ} \mathrm{C}$, the reaction was quenched by the addition of a $2 \mathrm{~mL} \mathrm{1:1} \mathrm{mixture} \mathrm{of} \mathrm{sat.} \mathrm{aqueous} \mathrm{Na}_{2} \mathrm{~S}_{2} \mathrm{O}_{3}$ and $\mathrm{NaHCO}_{3}$, 
followed by warming to room temperature and diluting with EtOAc $(20 \mathrm{~mL})$. The layers were separated, and the organic layer was washed with sat. aqueous $\mathrm{NH}_{4} \mathrm{Cl}$, dried $\left(\mathrm{Na}_{2} \mathrm{SO}_{4}\right)$, filtered and concentrated. The residue was purified by PTLC (15:1, hexanes/EtOAc) to cyclic alkyne 16 as colorless oil (8.9 mg, 69\%). ${ }^{1} \mathrm{H}$ NMR $\left(\mathrm{CDCl}_{3}, 400 \mathrm{MHz}\right) \delta 6.70(\mathrm{dd}, J=15.6,11.1 \mathrm{~Hz}, 1 \mathrm{H}), 5.98(\mathrm{t}, J=10.8 \mathrm{~Hz}, 1 \mathrm{H}), 5.51$ $(\mathrm{dd}, J=15.6,7.7 \mathrm{~Hz}, 1 \mathrm{H}), 5.20-5.14(\mathrm{~m}, 1 \mathrm{H}), 4.92(\mathrm{t}, J=10.7 \mathrm{~Hz}, 1 \mathrm{H}), 4.38-4.33(\mathrm{~m}, 1 \mathrm{H}), 2.59$ (dd, $J=$ 16.8, $4.3 \mathrm{~Hz}, 1 \mathrm{H}), 2.41(\mathrm{dd}, J=16.7,10.9 \mathrm{~Hz}, 1 \mathrm{H}), 2.07(\mathrm{dt}, J=14.8,1.8 \mathrm{~Hz}, 1 \mathrm{H}), 1.74-1.67(\mathrm{~m}, 1 \mathrm{H})$, $1.33(\mathrm{~d}, J=6.5 \mathrm{~Hz}, 3 \mathrm{H}), 1.19-1.12(\mathrm{~m}, 1 \mathrm{H}), 0.93-0.87(\mathrm{~m}, 1 \mathrm{H}), 0.89(\mathrm{~s}, 9 \mathrm{H}), 0.69-0.65(\mathrm{~m}, 1 \mathrm{H}), 0.63-$ $0.58(\mathrm{~m}, 1 \mathrm{H}), 0.07(\mathrm{~s}, 6 \mathrm{H}) ;{ }^{13} \mathrm{C} \mathrm{NMR}\left(\mathrm{CDCl}_{3}, 100 \mathrm{~Hz}\right) \delta 153.4,136.5,132.5,128.3,125.4,85.7,77.4$ 73.8, 72.0, 38.6, 29.2, 26.0, 20.4, 18.4, 18.2, 14.6, 12.8; IR (film) $v_{\max } 2954,2928,2856,2238,1706$, 1252, 1105, 1072, 1004; ESIMS $m / z 383\left(\left[\mathrm{M}+\mathrm{Na}^{+}\right], \mathrm{C}_{21} \mathrm{H}_{32} \mathrm{NaO}_{3}\right.$ Si requires 383); HRMS (FAB $\left.{ }^{+}\right) m / z$ $360.2130\left([\mathrm{M}]^{+}, \mathrm{C}_{21} \mathrm{H}_{32} \mathrm{O}_{3}\right.$ Si requires 360.2121). $[\alpha]^{25}+56\left(c 0.95, \mathrm{CHCl}_{3}\right)$.

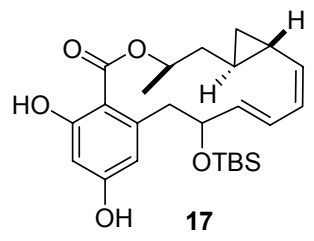

Diels-Alder product 17. Compound $16(156 \mathrm{mg}, 0.43 \mathrm{mmol})$ was dissolved in diene $12(0.75 \mathrm{~mL}, 2.3$ mmol) and heated to $140{ }^{\circ} \mathrm{C}$ in a sealed vial for 66 hours. The reaction mixture was cooled to room temperature and purified by PTLC (4:1, hexanes/EtOAc) to give $17(143 \mathrm{mg}, 75 \%) .{ }^{1} \mathrm{H} \mathrm{NMR}\left(\mathrm{CDCl}_{3}\right.$, $400 \mathrm{MHz}) \delta 11.58(\mathrm{~s}, 1 \mathrm{H}), 6.56(\mathrm{dd}, J=15.9,9.3 \mathrm{~Hz}, 1 \mathrm{H}), 6.38(\mathrm{~d}, J=2.4 \mathrm{~Hz}, 1 \mathrm{H}), 6.33(\mathrm{~d}, J=2.4 \mathrm{~Hz}$ $1 \mathrm{H}), 5.94(\mathrm{t}, J=9.5 \mathrm{~Hz}, 1 \mathrm{H}), 5.68(\mathrm{dd}, J=15.9,6.8 \mathrm{~Hz}, 1 \mathrm{H}), 5.46-5.43(\mathrm{~m}, 1 \mathrm{H}), 5.34(\mathrm{dd}, J=10.1,4.3$ $\mathrm{Hz}, 1 \mathrm{H}), 4.51(\mathrm{q}, J=6.7 \mathrm{~Hz}, 1 \mathrm{H}), 3.79(\mathrm{dd}, J=13.6,6.2 \mathrm{~Hz}, 1 \mathrm{H}), 2.81(\mathrm{dd}, J=13.1,8.9 \mathrm{~Hz}, 1 \mathrm{H}), 1.99-$ $1.81(\mathrm{~m}, 2 \mathrm{H}), 1.44(\mathrm{~d}, J=6.5 \mathrm{~Hz}, 3 \mathrm{H}), 1.21-1.13(\mathrm{~m}, 1 \mathrm{H}), 0.93-0.87(\mathrm{~m}, 1 \mathrm{H}), 0.88(\mathrm{~s}, 9 \mathrm{H}), 0.58-0.51(\mathrm{~m}$ 2H), -0.02 (s, 6H); ${ }^{13} \mathrm{C} \mathrm{NMR}\left(\mathrm{CDCl}_{3}, 100 \mathrm{~Hz}\right) \delta 164.8,160.5,143.8,134.8,133.7,129.6,128.0,112.3$, 106.1, 102.1, 75.7, 72.9, 42.6, 38.0, 26.1, 18.7, 18.4, 16.4, 16.1, 14.8, 1.4, -4.4, -4.6; IR (film) $v_{\max } 3376$, 
2954, 2928, 2856, 1644, 1619, 1257, 1062, 835; ESIMS $m / z 467\left(\left[\mathrm{M}+\mathrm{Na}^{+}\right], \mathrm{C}_{25} \mathrm{H}_{36} \mathrm{NaO}_{5} \mathrm{Si}\right.$ requires 467). HRMS $\left(\mathrm{FAB}^{+}\right) \mathrm{m} / \mathrm{z} 444.2336\left([\mathrm{M}]^{+}, \mathrm{C}_{25} \mathrm{H}_{36} \mathrm{O}_{5}\right.$ Si requires 444.2332). $[\alpha]^{25}{ }_{\mathrm{D}}+3.1\left(c 1.3, \mathrm{CHCl}_{3}\right)$.

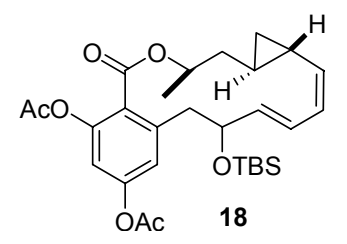

To a solution of 17 (243 mg, $0.546 \mathrm{mmol})$ in anhydrous DMF (13.5 mL) was added acetic anhydride $(2.9 \mathrm{~mL})$ and $4-($ dimethylamino)pyridine $(12.0 \mathrm{mg}, 0.0546 \mathrm{mmol})$ sequentially. After $30 \mathrm{~min}$ at room temperature, the reaction was quenched by addition of $50 \mathrm{~mL}$ of $\mathrm{pH} 7.2$ phosphate buffer. The resulting mixture was diluted with EtOAc $(75 \mathrm{~mL})$, separated and the aqueous layer was extracted with additional EtOAc $(2 \times 50 \mathrm{~mL})$. The combined organic layers were washed with $5 \%$ aqueous $\mathrm{NaCl}(2 \times 45 \mathrm{~mL})$. The combined washings were extracted with EtOAc $(2$ x $30 \mathrm{~mL})$. The combined organic layers were washed with sat. aqueous $\mathrm{NaCl}(30 \mathrm{~mL})$, dried $\left(\mathrm{Na}_{2} \mathrm{SO}_{4}\right)$, filtered and concentrated. Purification of the residue by PTLC (4:1 hexane/EtOAc) gave diacetate $18(250 \mathrm{mg}, 87 \%) .{ }^{1} \mathrm{H} \mathrm{NMR}\left(\mathrm{CDCl}_{3}, 400 \mathrm{MHz}\right) \delta$ $6.96(\mathrm{~d}, J=2.1 \mathrm{~Hz}, 1 \mathrm{H}), 6.88(\mathrm{~d}, J=2.1 \mathrm{~Hz}, 1 \mathrm{H}), 6.59(\mathrm{dd}, J=16.0,10.5 \mathrm{~Hz}, 1 \mathrm{H}), 5.89(\mathrm{t}, J=10.5 \mathrm{~Hz}$ $1 \mathrm{H}), 5.66(\mathrm{dd}, J=16.1,4.9 \mathrm{~Hz}, 1 \mathrm{H}), 5.26-5.23(\mathrm{~m}, 2 \mathrm{H}), 4.51(\mathrm{q}, J=5.8 \mathrm{~Hz}, 1 \mathrm{H}), 3.12-3.02(\mathrm{~m}, 2 \mathrm{H})$, $2.28,2.23(2 \mathrm{~s}, 6 \mathrm{H}), 1.47(\mathrm{~d}, J=6.2 \mathrm{~Hz}, 3 \mathrm{H}), 1.46-1.39(\mathrm{~m}, 1 \mathrm{H}), 1.14(\mathrm{ddd}, J=14.9,10.1,2.0 \mathrm{~Hz}, 1 \mathrm{H})$, 0.96-0.87 (m, 1H), $0.87(\mathrm{~s}, 9 \mathrm{H}), 0.52-0.48(\mathrm{~m}, 2 \mathrm{H}),-0.01,-0.02(2 \mathrm{~s}, 6 \mathrm{H}) ;{ }^{13} \mathrm{C} \mathrm{NMR}\left(\mathrm{CDCl}_{3}, 100 \mathrm{~Hz}\right) \delta$ $168.6,168.4,166.4,151.4,148.7,139.1,135.9,133.6,129.2,127.2,125.6,120.4,114.7,73.8,73.2$ $43.2,40.0,26.0,21.3,21.0,19.3,18.3,16.9,16.2,17.7,-4.6,-4.7$; IR (film) $v_{\max } 2954,2928,2856$, 1775, 1720, 1612, 1191, 1134, 1069; ESIMS $m / z 551\left(\left[\mathrm{M}+\mathrm{Na}^{+}\right], \mathrm{C}_{29} \mathrm{H}_{40} \mathrm{NaO}_{7} \mathrm{Si}\right.$ requires 551); HRMS $\left(\mathrm{FAB}^{+}\right) m / z 528.2569\left([\mathrm{M}]^{+}, \mathrm{C}_{29} \mathrm{H}_{40} \mathrm{O}_{7} \mathrm{Si}\right.$ requires 528.2543). $[\alpha]^{25}-38\left(c 1.1, \mathrm{CHCl}_{3}\right)$.

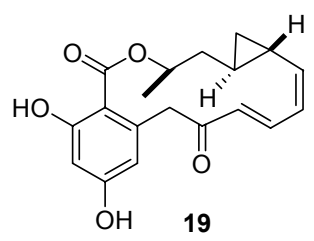


Cyclopropamonocillin 19. To a solution of diacetate $18(250 \mathrm{mg}, 0.473 \mathrm{mmol})$ in $\mathrm{THF}(10.2 \mathrm{~mL})$ at $0{ }^{\circ} \mathrm{C}$ was added pyridine $(3.4 \mathrm{~mL})$ and HF-Pyridine complex $(1.7 \mathrm{~mL})$ sequentially. The resulting mixture was stirred at room temperature for $10.5 \mathrm{hrs}$. TMSOMe $(30 \mathrm{~mL})$ was added, and stirring was continued for $45 \mathrm{~min}$ to quench the remaining HF. The solvents were removed under reduced pressure. The alcohol isolated was dried under high vacuum, and dissolved in $\mathrm{CH}_{2} \mathrm{Cl}_{2}(15 \mathrm{~mL})$ and cooled to $0{ }^{\circ} \mathrm{C}$. DessMartin periodinane $(301 \mathrm{mg}, 0.710 \mathrm{mmol})$ was added, and the resulting mixture was stirred at room temperature for $15 \mathrm{~min}$. The solution was then directly loaded on PTLC plates and purified (1:1 hexane/EtOAc) to give the desired ketone $(133 \mathrm{mg}, 68 \%) .{ }^{1} \mathrm{H} \mathrm{NMR}\left(\mathrm{CDCl}_{3}, 500 \mathrm{MHz}\right) \delta 8.01(\mathrm{dd}, J=$ 16.1, 11.3 Hz, 1H), $6.97(\mathrm{~d}, J=1.4 \mathrm{~Hz}, 1 \mathrm{H}), 6.92(\mathrm{~d}, J=2.0 \mathrm{~Hz}, 1 \mathrm{H}), 6.20(\mathrm{t}, J=10.4 \mathrm{~Hz}, 1 \mathrm{H}), 5.96(\mathrm{~d}$, $J=16.0 \mathrm{~Hz}, 1 \mathrm{H}), 5.60(\mathrm{dd}, J=10.0,7.2 \mathrm{~Hz}, 1 \mathrm{H}), 5.47-5.41(\mathrm{~m}, 1 \mathrm{H}), 4.20(\mathrm{~d}, J=13.8 \mathrm{~Hz}, 1 \mathrm{H}), 3.77(\mathrm{~d}$, $J=13.8 \mathrm{~Hz}, 1 \mathrm{H}), 2.31(\mathrm{dt}, J=15.3,4.4 \mathrm{~Hz}, 1 \mathrm{H}), 2.25,2.24(2 \mathrm{~s}, 6 \mathrm{H}), 1.73-1.71(\mathrm{~m}, 1 \mathrm{H}), 1.50(\mathrm{~d}, J=6.5$ $\mathrm{Hz}, 3 \mathrm{H}), 1.26-1.21(\mathrm{~m}, 1 \mathrm{H}), 1.00-0.97(\mathrm{~m}, 1 \mathrm{H}), 0.75-0.69(\mathrm{~m}, 2 \mathrm{H}) ;{ }^{13} \mathrm{C} \mathrm{NMR}\left(\mathrm{CDCl}_{3}, 125 \mathrm{~Hz}\right) \delta 198.4$ $168.5,165.0,152.1,149.2,145.6,143.7,135.7,129.6,128.8,124.6,119.1,115.6,72.8,43.0,38.3,21.2$ 18.2, 16.7, 16.5, 15.6; IR (film) $v_{\max } 2928,1774,1728,1657,1621,1586,1290,1190,1132,1029$; ESIMS $m / z 435\left(\left[\mathrm{M}+\mathrm{Na}^{+}\right], \mathrm{C}_{23} \mathrm{H}_{24} \mathrm{NaO}_{7}\right.$ requires 435); HRMS $\left(\mathrm{FAB}^{+}\right) m / z 413.1616\left(\left[\mathrm{M}+\mathrm{H}^{+}\right.\right.$, $\mathrm{C}_{23} \mathrm{H}_{25} \mathrm{O}_{7}$ requires 413.1600). [ $\left.\alpha\right]^{25}-269$ (c 1.43, $\left.\mathrm{CHCl}_{3}\right)$.

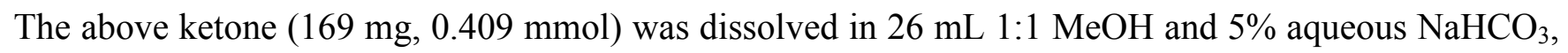
and stirred at room temperature for $14 \mathrm{hrs}$ to remove the phenolic acetates. The resulting solution was diluted with sat. aqueous $\mathrm{NH}_{4} \mathrm{Cl}(80 \mathrm{~mL})$ and EtOAc $(100 \mathrm{~mL})$ and separated. The aqueous layer was extracted with additional EtOAc $(3 \times 75 \mathrm{~mL})$. The combined organic layers were dried $\left(\mathrm{Na}_{2} \mathrm{SO}_{4}\right)$, filtered and concentrated in vacuum. Purification by PTLC (1:1 hexane/EtOAc) provided 19 (124 mg, 92\%). ${ }^{1} \mathrm{H} \mathrm{NMR}\left(\mathrm{CDCl}_{3}, 500 \mathrm{MHz}\right) \delta 11.33(\mathrm{~s}, 1 \mathrm{H}), 8.36(\mathrm{dd}, J=16.1,11.7 \mathrm{~Hz}, 1 \mathrm{H}), 6.48(\mathrm{~d}, J=1.6 \mathrm{~Hz}$ $1 \mathrm{H}), 6.40(\mathrm{~d}, J=2.5 \mathrm{~Hz}, 1 \mathrm{H}), 6.20(\mathrm{t}, J=11.3 \mathrm{~Hz}, 1 \mathrm{H}), 6.01(\mathrm{~d}, J=15.9 \mathrm{~Hz}, 1 \mathrm{H}), 5.72(\mathrm{dd}, J=9.8,6.2$ $\mathrm{Hz}, 1 \mathrm{H}), 5.49-5.45(\mathrm{~m}, 1 \mathrm{H}), 5.32(\mathrm{~d}, J=13.6 \mathrm{~Hz}, 1 \mathrm{H}), 3.51(\mathrm{~d}, J=13.7 \mathrm{~Hz}, 1 \mathrm{H}), 2.31(\mathrm{dt}, J=15.8,3.3$ $\mathrm{Hz}, 1 \mathrm{H}), 1.61-1.55(\mathrm{~m}, 1 \mathrm{H}), 1.55(\mathrm{~d}, J=6.7 \mathrm{~Hz}, 3 \mathrm{H}), 1.32-1.28(\mathrm{~m}, 1 \mathrm{H}), 1.03-0.97(\mathrm{~m}, 1 \mathrm{H}), 0.74-0.72$ 
$(\mathrm{m}, 1 \mathrm{H}), 0.64-0.63(\mathrm{~m}, 1 \mathrm{H}) ;{ }^{13} \mathrm{C} \mathrm{NMR}\left(\mathrm{CDCl}_{3}, 125 \mathrm{~Hz}\right) \delta 201.8,170.1,165.6,161.6,145.3,139.2$ $129.5,128.5,109.4,104.5,102.7,73.5,43.2,27.6,17.9,17.2,15.9,13.9$; IR (film) $v_{\max } 3260,1650$ 1618, 1586, 1447, 1259, 1160, 1099, 996, 854; ESIMS $m / z 351\left(\left[\mathrm{M}+\mathrm{Na}^{+}\right], \mathrm{C}_{19} \mathrm{H}_{20} \mathrm{NaO}_{5}\right.$ requires 351); HRMS $\left(\mathrm{FAB}^{+}\right) m / z 329.1382\left([\mathrm{M}+\mathrm{H}]^{+}, \mathrm{C}_{19} \mathrm{H}_{21} \mathrm{O}_{5}\right.$ requires 329.1389). $(+)-(R, R, S)-19:[\alpha]^{25}{ }_{\mathrm{D}}-189(c$ $\left.0.77, \mathrm{CH}_{2} \mathrm{Cl}_{2}\right)$.

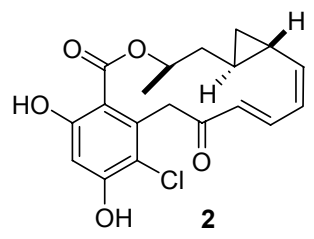

Cycloproparadicicol 2. Compound 19 (26.5 mg, $0.081 \mathrm{mmol})$ was dissolved in dry $\mathrm{CH}_{2} \mathrm{Cl}_{2}(5 \mathrm{~mL})$ and cooled to $0{ }^{\circ} \mathrm{C}$. A solution of $\mathrm{SO}_{2} \mathrm{Cl}_{2}$ in $\mathrm{CH}_{2} \mathrm{Cl}_{2}\left(4.5 \mathrm{~mL}\right.$, diluted from $0.123 \mathrm{~mL} 1 \mathrm{M}$ solution in $\mathrm{CH}_{2} \mathrm{Cl}_{2}$, $0.123 \mathrm{mmol}$ ) was added dropwise. After 45 minutes, the reaction was quenched by the addition of $5 \mathrm{~mL}$ of $5 \% \mathrm{NH}_{4} \mathrm{Cl}$, and diluted with $\mathrm{CH}_{2} \mathrm{Cl}_{2}$. The layers were separated, and the organic phase was washed with brine, dried $\left(\mathrm{MgSO}_{4}\right)$, filtered and concentrated in vacuum. Purification of the residue by PTLC (3:1, hexanes/EtOAc) gave 2 as a white solid (17.8 mg, 61\%). ${ }^{1} \mathrm{H} \mathrm{NMR}\left(\mathrm{CDCl}_{3}, 500 \mathrm{MHz}\right) \delta 10.89(\mathrm{~s}$, $1 \mathrm{H}), 8.00(\mathrm{dd}, J=15.9,11.3 \mathrm{~Hz}, 1 \mathrm{H}), 6.63(\mathrm{~s}, 1 \mathrm{H}), 6.42(\mathrm{~s}, 1 \mathrm{H}), 6.13(\mathrm{t}, J=11.2 \mathrm{~Hz}, 1 \mathrm{H}), 6.04(\mathrm{~d}, J=$ $16.3 \mathrm{~Hz}, 1 \mathrm{H}), 5.62(\mathrm{dd}, J=9.9,5.8 \mathrm{~Hz}, 1 \mathrm{H}), 5.46-5.42(\mathrm{~m}, 1 \mathrm{H}), 4.90($ broad d, $J=15.9 \mathrm{~Hz}, 1 \mathrm{H}), 3.77$ (bs, $1 \mathrm{H}), 2.22(\mathrm{dt}, J=15.8,3.2 \mathrm{~Hz}, 1 \mathrm{H}), 1.60-1.52(\mathrm{~m}, 1 \mathrm{H}), 1.48(\mathrm{~d}, J=6.7 \mathrm{~Hz}, 3 \mathrm{H}), 1.15-1.11(\mathrm{~m}, 1 \mathrm{H})$, 0.90-0.85 (m, 1H), 0.69-0.65 (m, 1H), 0.57-0.54 (m, 1H); ${ }^{13} \mathrm{C}$ NMR $\left(\mathrm{CDCl}_{3}, 125 \mathrm{~Hz}\right) \delta 199.2,169.4$ $162.7,155.6,143.5,142.5,136.9,129.9,128.9,115.6,107.8,103.5,74.4,46.4,37.4,17.9,17.3,15.6$ 13.6; IR (film) $v_{\max } 3341,1716,1651,1609,1578 \mathrm{~cm}^{-1}$; ESIMS $m / z 385\left(\left[\mathrm{M}+\mathrm{Na}^{+}\right], \mathrm{C}_{19} \mathrm{H}_{19} \mathrm{NaO}_{5} \mathrm{Cl}\right.$ requires 385); HRMS (ESI) $m / z 385.0820\left(\left[\mathrm{M}+\mathrm{Na}^{+}\right], \mathrm{C}_{19} \mathrm{H}_{19} \mathrm{NaO}_{5} \mathrm{Cl}\right.$ requires 385.0819). (+)-(R, $\left.R, S\right)$ 2: $[\alpha]^{25}+69\left(c 0.87, \mathrm{CH}_{2} \mathrm{Cl}_{2}\right)$. 